\title{
DERIVADOS ORGANOMETÁlICOS DE ESTANHO(II) - PARTE 1. COMPOSTOS CICLOPENTADIENÍLICOS
}

Geraldo M. de Lima

Depto de Química, Universidade Federal de Minas Gerais, 31270-901 Belo Horizonte - MG

Recebido em 27/3/00; aceito em 17/11/00

\begin{abstract}
ORGANOMETALLIC TIN(II) DERIVATIVES - PART 1. CYCLOPENTADIENYL COMPOUNDS. This paper provides a brief review of the chemistry of cyclopentadienyl Sn(II) derivatives which includes the preparation, the molecular structure and reactivity associated with such bis-sandwich tin(II) species. It is compared structural and spectroscopic results and it is also discussed how the nature of the cyclopentadienyl ring bonded to the Sn centre plays an important role in the structural and stability features of the derivatives. Bulk rings such as $\mathrm{C}_{5} \mathrm{HPr}_{4}{ }_{4}^{-}, \mathrm{C}_{5} \mathrm{Bz}_{5}{ }^{-}, \mathrm{C}_{5} \mathrm{Me}_{4} \mathrm{SiMe}_{2} \mathrm{Bu}^{\mathrm{t}-}$ and $\mathrm{C}_{5} \mathrm{Ph}_{5}^{-}$render airstable and parallel ring-bonded compounds.
\end{abstract}

Keywords: organometallic tin(II) compounds; solid state NMR; cyclopentadienyl tin(II) derivatives.

\section{INTRODUÇÃO}

Espécies organometálicas de $\mathrm{Sn}(\mathrm{II})$, os chamados estanilenos de forma geral $\mathrm{SnR}_{2}$, eram conhecidos pelo menos 140 anos antes da preparação de $\left[\mathrm{Sn}\left(\eta^{5}-\mathrm{C}_{5} \mathrm{H}_{5}\right)_{2}\right]^{1}$. Entretanto, muitas das espécies descritas naquela época eram de fato intermediários bastante reativos, os quais se apresentavam na forma de $\mathrm{SnR}_{2}$ somente na fase gasosa. Uma vez no estado sólido as unidades $\mathrm{SnR}_{2}$ se agrupavam na forma de oligômeros ou polímeros de formulação geral $\left(\mathrm{R}_{2} \mathrm{Sn}\right)_{\mathrm{n}}{ }^{2}$. Para manter a espécie na forma monomérica $\left\{\mathrm{SnR}_{2}\right\}$ e evitar polimerização passou-se a utilizar grupos orgânicos, R, com um elevado impedimento estérico. As espécies geradas a partir deste processo constituíram uma nova classe de compostos de estanho. No entanto, esta classe passou a ser dividida em duas famílias distintas de compostos de $\mathrm{Sn}$ (II), aqueles que apresentam ligações $\mathrm{Sn}-\mathrm{C}$ do tipo $\pi$ ou seja os ciclopentadienílicos e a segunda classe, a daqueles compostos cuja ligação $\mathrm{Sn}-\mathrm{C}$ é de natureza $\sigma$. Os últimos são análogos aos carbenos: $\mathrm{CR}_{2}$, enquanto os primeiros não possuem equivalência na química orgânica. Dentro da classe dos compostos cuja ligação Sn-C é do tipo $\sigma$ existem ainda os estanatos de formulação $\mathrm{SnR}_{3}{ }^{-}$. Neste artigo serão abordados apenas aspectos relacionados com compostos ciclopentadienílicos de $\mathrm{Sn}(\mathrm{II})$.

\section{DERIVADOS CICLOPENTADIENÍLICOS DO ESTANHO(II)}

\section{Propriedades principais}

Devido à suas propriedades estéricas e eletrônicas, ligantes ciclopentadienílicos podem ser apontados como dos mais importantes na química organometálica. Uma vez que o estanho se encontra ligado aos átomos de carbono do anel $\mathrm{Cp}$ via ligação $\pi$, em analogia ao ferroceno, compostos de $\mathrm{Sn}$ (II) contendo este tipo de ligantes são denominados de estanocenos. Tais compostos são comumente preparados a partir da reação de $\mathrm{SnX}_{2}(\mathrm{X}=\mathrm{Cl}, \mathrm{Br}$ ou I) com o organolítio ou reagentes de Grignard $\left\{\mathrm{LiCp}\right.$ ou $\left.\mathrm{MgCp}_{2}\right\}$ apropriados, numa temperatura de $-78^{\circ} \mathrm{C}$, em atmosfera inerte e utilizando-se $\mathrm{Et}_{2} \mathrm{O}$ como solvente. Eles são isolados como sólidos sensíveis à umidade e ao oxigênio, podem apresentar coloração que varia de amarelo ouro até incolor. Todos os estanocenos são sensíveis à umidade e ao ar, exceto $\left[\operatorname{Sn}\left(\eta^{5}-\mathrm{C}_{5} \mathrm{Ph}_{5}\right)_{2}\right],\left[\operatorname{Sn}\left(\eta^{5}-\right.\right.$ $\left.\left.\mathrm{C}_{5} \mathrm{Bz}_{5}\right)_{2}\right]^{4}, \quad\left[\mathrm{Sn}\left(\eta^{5} \mathrm{C}_{5} \mathrm{Pr}^{\mathrm{i}}{ }_{4} \mathrm{H}\right)_{2}\right]$ e $\left[\mathrm{Sn}\left(\eta^{5}-\mathrm{Cp}^{\mathrm{S}}\right)_{2}\right]$.

As propriedades estruturais e estabilidade dos estanocenos dependem fortemente da natureza do grupo $\mathrm{Cp}$. Na maioria deles os anéis carbônicos se apresentam de forma não-paralela, $\left[\operatorname{Sn}\left(\eta^{5}-\right.\right.$ $\left.\mathrm{C}_{5} \mathrm{H}_{5}\right)^{2}$ ] (Figura 1), [ $\left.\mathrm{Sn}\left(\eta^{5}-\mathrm{C}_{5} \mathrm{Me}_{5}\right)_{2}\right]$.
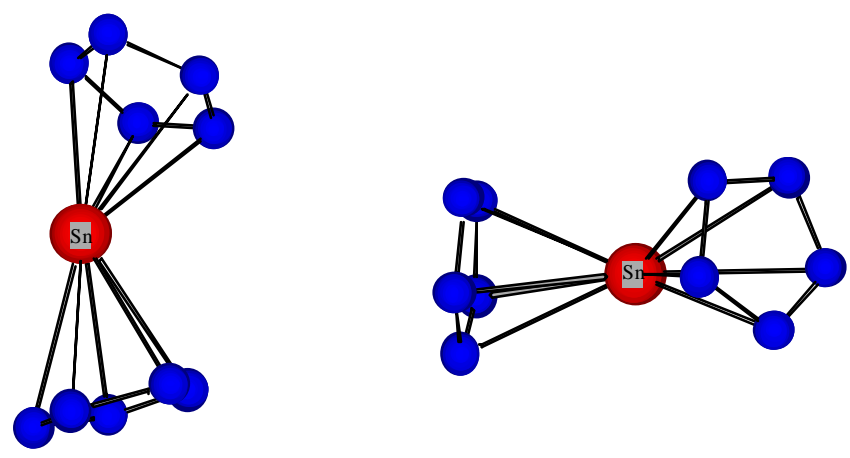

Figura 1. Estrutura molecular do $\left[\mathrm{Sn}\left(\eta^{5}-\mathrm{C}_{5} \mathrm{H}_{5}\right)_{2}\right]$.

Neste caso o par de elétrons livre se localiza num orbital molecular com forte caráter $\mathrm{sp}^{2}$. Em contrapartida, existem alguns exemplos de compostos onde se observa um arranjo paralelo dos anéis (Figura 2) onde o par de elétrons se localiza num orbital com um acentuado caráter $s$. Isto acontece quando se introduz substituintes volumosos nos anéis $\mathrm{Cp}$. A presença de grupos "Cp" volumosos ligados ao $\mathrm{Sn}$ (II) protege o centro metálico de ataques externos \{oxidação, hidrólise, etc\}, desta forma certos estanocenos são estáveis ao ar ou umidade. Ainda não existe uma explicação clara para o fato de os estanocenos optarem por uma estrutura com anéis paralelos ou não paralelos ${ }^{8}$.
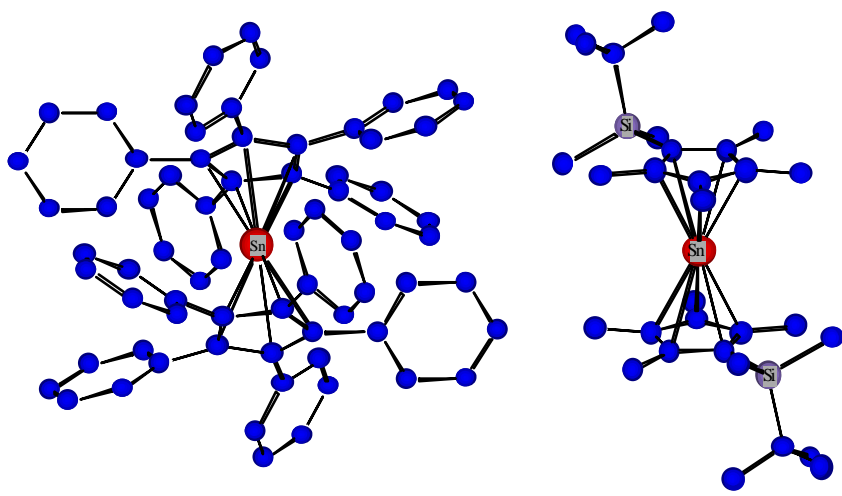

Figura 2. Estruturas moleculares de $\left[\operatorname{Sn}\left(\boldsymbol{\eta}^{5}-C_{5} P h_{5}\right)_{2}\right]^{9}$ e $\left[\operatorname{Sn}\left(\boldsymbol{\eta}^{5}-\right.\right.$ $\left.\left(p^{S}\right)_{2}\right]^{10}$. 
Todos os estanocenos apresentam uma assimetria nas interações Sn-Cp implicando em diferenças bastante substanciais nas ligações Sn-C que podem variar entre 2,53 e 2,85 A (Tabela 1). Entretanto tal assimetria é observada apenas em experimentos de difração de raios-X. Estudos de ressonância magnética nuclear de ${ }^{13} \mathrm{C}$ utilizando-se, por exemplo, $\left[\mathrm{Sn}\left(\eta^{5}-\mathrm{C}_{5} \mathrm{H}_{5}\right)_{2}\right]$ não foram capazes de revelar diferença química nas interações $\mathrm{Sn}-\mathrm{C}$, em virtude de se obter apenas um sinal no espectro, mesmo à baixa temperatura. Observandose ainda na Tabela 1, verifica-se que as diferenças estruturais nas ligações Sn-C não causam grandes mudanças nos deslocamentos químicos de ${ }^{119} \mathrm{Sn}$ os quais variam de $\delta-2100$ até -2200 . Tal variação é muito pequena em se tratando de $\mathrm{Sn}(\mathrm{II})$.

No composto $\left[\mathrm{Sn}\left\{\eta^{5}-\left(\mathrm{Cp}^{\mathrm{B}}\right) \mathrm{Co}\left(\mathrm{C}_{5} \mathrm{H}_{5}\right)\right\}_{2}\right]^{11}$, (Figura 3) tais distâncias variam de 2,41 a 2,96 ̊ (Tabela 1).

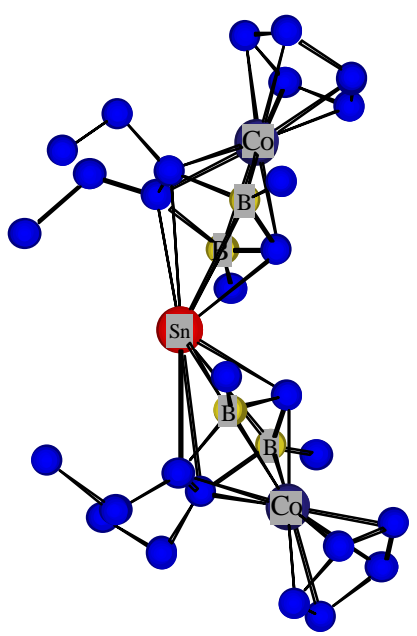

Figura 3. Estrutura molecular de $\left[\operatorname{Sn}\left\{\eta^{5}-\left(\mathrm{Cp}^{B}\right) \mathrm{Co}\left(\mathrm{C}_{5} \mathrm{H}_{5}\right)\right\}_{2}\right]$.

Em alguns heteroestanocenos, $\left[\operatorname{Sn}\left\{\eta^{5}-\mathrm{Cp}^{\mathrm{BN}}\right\}_{2}\right]^{12}$ e $\left[\operatorname{Sn}\left\{\eta^{5}-\right.\right.$ $\left.\left.C p^{N}\right\}^{2}\right]^{13}$, (Figura 4), as ligações Sn-C são ainda mais diferentes. As distâncias Sn-N, 2,917(9) no primeiro e 2,943(5) ̊ no segundo são as mais longas.

Como se deveria esperar as propriedades físicas, estruturais e reatividade dos estanocenos são bastante semelhantes às dos outros metalocenos do grupo 14 da tabela periódica $\{\mathrm{Si}(\mathrm{II})$, $\mathrm{Ge}(\mathrm{II})$ e $\mathrm{Pb}(\mathrm{II})\}$. Entretanto, observa-se grande diferença entre as características destes compostos com as daqueles metalocenos de elementos de transição. Embora a estratégia de síntese seja semelhante, ao se comparar por exemplo $\left[\operatorname{Sn}\left(\eta^{5}\right.\right.$ $\left.\left.\mathrm{C}_{5} \mathrm{H}_{5}\right)_{2}\right]$ com $\left[\mathrm{Fe}\left(\eta^{5}-\mathrm{C}_{5} \mathrm{H}_{5}\right)_{2}\right]$ observa-se pouca similaridade
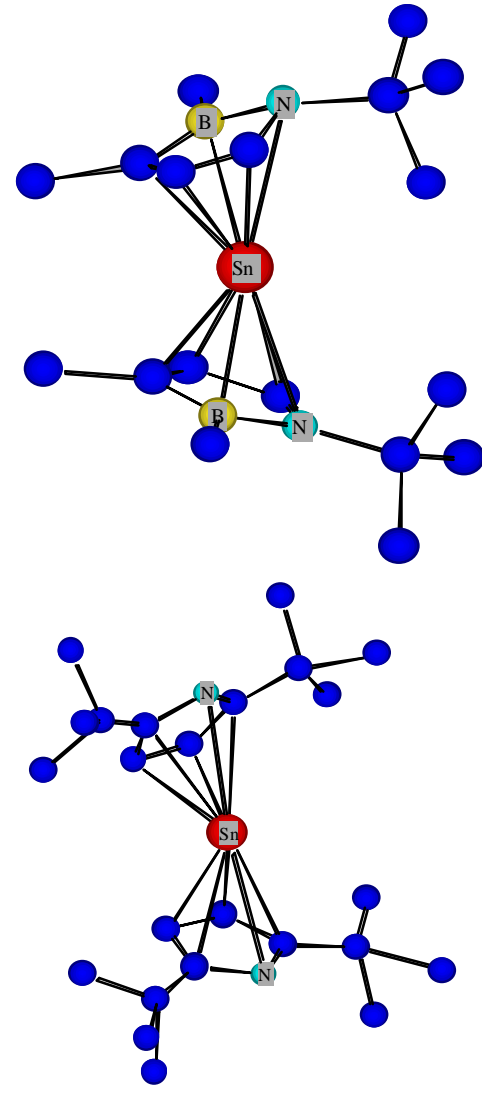

Figura 4. Estrutura molecular dos compostos $\left[S n\left\{\eta^{5}-C p^{B N}\right\}_{2}\right]$ e $\left[\operatorname{Sn}\left\{\boldsymbol{\eta}^{5}-C p^{N}\right\}_{2}\right]$.

entre

estes compostos. A primeira diferença é o fato do $\left[\mathrm{Fe}\left(\eta^{5}-\mathrm{C}_{5} \mathrm{H}_{5}\right)_{2}\right]$ possuir 18 elétrons de valência enquanto o estanoceno é uma espécie deficiente em elétrons. O $\mathrm{Sn}(\mathrm{II})$ se encontra mais afastado do centróide do anel $\mathrm{Cp}, 2,4 \AA$, do que o Fe(II), 1,66 $⿱$, refletindo o fato de as ligações $\mathrm{Fe}-\mathrm{C}$ serem mais fortes. Desta forma é muito difícil remover um dos anéis $\mathrm{Cp}$ do $\left[\mathrm{Fe}\left(\eta^{5}\right.\right.$ $\left.\mathrm{C}_{5} \mathrm{H}_{5}\right)_{2}$ ] \{objetivando a formação de espécies heterolépticas do tipo $\mathrm{Fe}\left(\eta^{5}-\mathrm{C}_{5} \mathrm{H}_{5}\right) \mathrm{R}$ \} enquanto que ocorre eliminação de $\mathrm{LiCp}$ ao se reagir $\left[\operatorname{Sn}\left(\eta^{5}-\mathrm{C}_{5} \mathrm{H}_{5}\right)_{2}\right]$ com algum organolítio \{ocorrendo a formação de $\left.\mathrm{Sn}\left(\eta^{5}-\mathrm{C}_{5} \mathrm{H}_{5}\right) \mathrm{R}\right\}$. As reações subsequentes do ferroceno se assemelham mais às dos hidrocarbonetos aromáticos \{Acilação de Friedel-Crafts, Aminometilação de Mannich e Metalações em geral\}, sendo que a cinética de muitas delas

Tabela 1. Dados cristalográficos e de RMN de ${ }^{119} \mathrm{Sn}$ em solução e no estado sólido selecionados para alguns estanocenos *

\begin{tabular}{|c|c|c|c|c|c|}
\hline Derivados & Distância Sn-M (Å) & Ângulo $\mathrm{M}-\mathrm{Sn}-\mathrm{M}\left({ }^{\mathrm{o}}\right)$ & $\mathrm{RMN}{ }^{119} \mathrm{Sn}(\delta)$ & ${ }^{119} \mathrm{Sn} \operatorname{MAS}\left(\delta_{\mathrm{iso}}\right)$ & Ref. \\
\hline$\left[\mathrm{Sn}\left(\eta^{5}-\mathrm{C}_{5} \mathrm{H}_{5}\right)_{2}\right]$ & $2,410(\mathrm{~m}), 2,.404(\mathrm{~m})$. & $143,7,148,0$ & -2199 & $-2163,-2224$ & 1 \\
\hline$\left[\mathrm{Sn}\left(\eta^{5}-\mathrm{Cp}^{*}\right)_{2}\right]$ & $2,450(\mathrm{~m}), 2,.434(\mathrm{~m})$. & $143,6,144,6$ & -2129 & $-2137,-2140$ & 6 \\
\hline$\left[\mathrm{Sn}\left\{\eta_{5}^{5}-\mathrm{C}_{5} \mathrm{H}_{4} \mathrm{P}\left[\mathrm{N}\left(\mathrm{Pr}^{\mathrm{i}}\right)_{2}\right]\right\}_{2}\right]$ & $2,383(\mathrm{~m})$. & 150,2 & - & - & 21 \\
\hline$\left[\mathrm{Sn}\left\{\eta^{5}-\mathrm{C}_{5} \mathrm{H}_{2}\left[\left(\mathrm{SiMe}_{3}\right)_{3}\right]\right\}_{2}\right]^{28}$ & $2,478(\mathrm{~m})$. & 162 & - & - & 28 \\
\hline$\left[\mathrm{Sn}\left\{\eta^{5}-\left(\mathrm{Cp}^{\mathrm{B}}\right) \operatorname{Co}\left(\mathrm{C}_{5} \mathrm{H}_{5}\right)\right\}_{2}\right]$ & $2,379(\mathrm{~m}), 2,.401(\mathrm{~m})$. & $141,6,142,6^{\mathrm{c}}$ & - & - & 11 \\
\hline$\left[\mathrm{Sn}\left(\eta^{5}-\mathrm{C}_{5} \mathrm{Ph}_{5}\right)_{2}\right]$ & $2,401(6)$ & 180 & - & -2215 & 9 \\
\hline$\left[\mathrm{Sn}\left(\eta^{5}-\mathrm{C}_{5} \mathrm{Ph}_{5}\right)\left(\eta-\mathrm{C}_{5} \mathrm{H}_{5}\right)\right]$ & $2,487(4), 2,391(5)^{a}$ & $151,1(1)$ & - & - & 9 \\
\hline$\left[\mathrm{Sn}\left(\eta^{5}-\mathrm{C}_{5} \mathrm{Bz}_{5}\right)_{2}\right]$ & $2,427(\mathrm{~m})$. & $155,9(9)$ & -2188 & -2288 & 4 \\
\hline$\left[\operatorname{Sn}\left\{\eta^{5}-\mathrm{Cp}^{\mathrm{BN}}\right\}_{2}\right]$ & $2,059(\mathrm{~m} .)^{b}$ & $160^{\mathrm{c}}$ & - & - & 12 \\
\hline$\left[\operatorname{Sn}\left\{\eta^{5}-\mathrm{Cp}^{\mathrm{N}}\right\}_{2}\right]$ & $2,428^{c}$ & $142,6^{\mathrm{c}}$ & -1889 & - & 13 \\
\hline$\left[\mathrm{Sn}\left(\eta^{5}-\mathrm{C}_{5} \mathrm{Pr}_{4}^{\mathrm{i}} \mathrm{H}\right)_{2}\right]$ & 2,424 & 165 & - & - & 5 \\
\hline$\left[\mathrm{Sn}\left(\eta^{5}-\mathrm{Cp}^{\mathrm{S}}\right)_{2}\right]$ & $2,379(2)$ & 180 & -2204 & -2236 & 10 \\
\hline
\end{tabular}

*Os deslocamentos químicos de ${ }^{119} \mathrm{Sn}$ são relativos a $\mathrm{SnMe}_{4}$; ${ }^{\mathrm{a}} \mathrm{Sn}-\mathrm{C}_{5} \mathrm{Ph}_{5}$ e $\mathrm{Sn}-\mathrm{C}_{5} \mathrm{H}_{5}$, respectivamente; ${ }^{\text {b }} \mathrm{Sn}-\mathrm{N}$ é muito longa; ${ }^{c}$ medido através de $\mathrm{C} 3 \mathrm{D}$; $\mathrm{M}$ denota o centróide do anel carbônico; $\mathrm{m}$. significa valor médio e $\delta_{\text {iso }}$ isotropical chemical shift. 


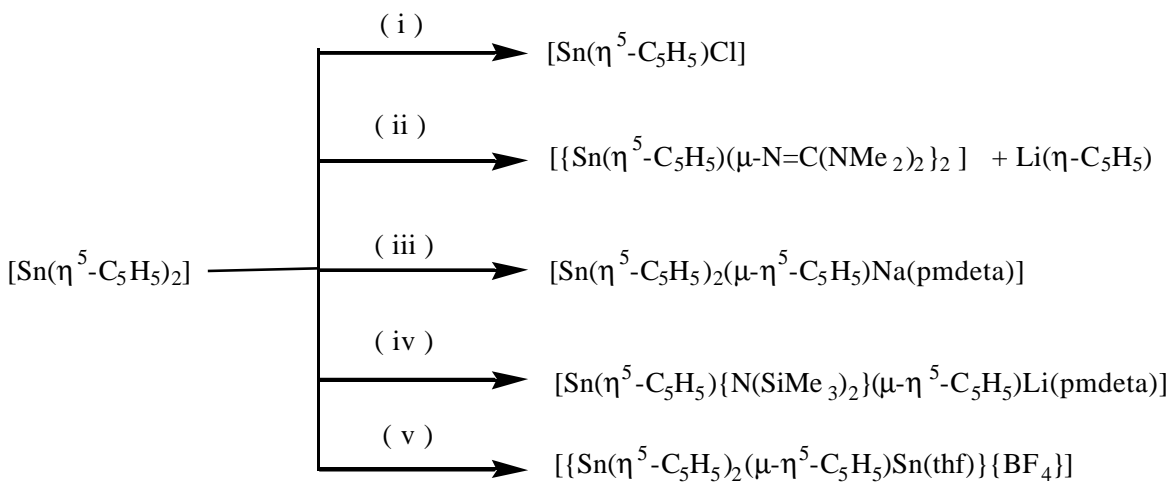

Condições:

(i) $\mathrm{SnCl}_{2} /$ thf; (ii) $\left.\mathrm{LiN}=\mathrm{C}\left(\mathrm{NMe}_{2}\right)_{2}\right) /$ thf; (iii) $\mathrm{Na}\left(\eta-\mathrm{C}_{5} \mathrm{H}_{5}\right) /$ pmdeta/thf; (iv)

(ii) $\mathrm{Li}\left\{\mathrm{N}\left(\mathrm{SiMe}_{3}\right)_{2}\right\} /$ pmedeta/thf; (v) $\mathrm{BF}_{3} /$ thf

Esquema 1. Reações do composto [ $\left.\operatorname{Sn}\left(\eta^{5}-C_{5} H_{5}\right)_{2}\right]$.

é mais rápida do que com o benzeno.

\section{REATIVIDADE}

Estanocenos podem ser utilizados como material de partida para outros compostos típicos. Derivados com características bastante interessantes podem ser obtidos ao se efetuar reações com alguns estanocenos (Esquema 1).

O derivado $\left[\mathrm{Sn}\left(\eta^{5}-\mathrm{C}_{5} \mathrm{H}_{5}\right) \mathrm{Cl}\right]$ é normalmente obtido ao se tratar [ $\mathrm{Sn}\left(\eta^{5}-\mathrm{C}_{5} \mathrm{H}_{5}\right)_{2}$ ] com $\mathrm{SnCl}_{2}$ em thf (i) ou então através da reação de $\mathrm{SnCl}_{2}$ com $\mathrm{Li}\left(\eta-\mathrm{C}_{5} \mathrm{H}_{5}\right)$ em $\mathrm{Et}_{2} \mathrm{O}$ na proporção de 1:1. Parece ser uma tendência geral todos os estanocenos gerarem compostos análogos através de reações similares. Este mesmo composto tem sido usado para fornecer outros derivados de $\mathrm{Sn}(\mathrm{II})$. Reagindo-se $\left[\mathrm{Sn}\left(\eta^{5}-\mathrm{C}_{5} \mathrm{H}_{5}\right) \mathrm{Cl}\right]$ com $\mathrm{Li}\left(\eta^{5}-\mathrm{C}_{5} \mathrm{Ph}_{5}\right)$ ou $\mathrm{KGe}\left(\mathrm{OBu}^{\mathrm{t}}\right)_{3}$ preparou-se $\left[\mathrm{Sn}\left(\eta^{5}-\mathrm{C}_{5} \mathrm{H}_{5}\right)\left(\eta^{5}-\mathrm{C}_{5} \mathrm{Ph}_{5}\right)\right]^{9}$ e $\left[\mathrm{Sn}\left(\eta^{5}-\mathrm{C}_{5} \mathrm{H}_{5}\right)\left(\mu-\mathrm{OBu}^{t}\right)_{2} \mathrm{Ge}\left(\mathrm{OBu}^{t}\right)\right]^{19}$, respectivamente. Estudos de $\mathrm{RMN}$ de ${ }^{119} \mathrm{Sn}$ para o derivado $\left[\mathrm{Sn}\left(\eta^{5}-\mathrm{C}_{5} \mathrm{H}_{5}\right)\left(\mu-\mathrm{OBu}^{\mathrm{t}}\right)_{2} \mathrm{Ge}\left(\mathrm{OBu}^{\mathrm{t}}\right)\right]$ revelaram uma drástica variação na forma de ligação do anel $\mathrm{Cp}$ em concordância com os parâmetros de raios-X. O deslocamento químico do ${ }^{119} \mathrm{Sn}$ sofre uma mudança bastante considerável variando de $\delta-1578$ no material de partida, $\left[\mathrm{Sn}\left(\eta^{5}-\mathrm{C}_{5} \mathrm{H}_{5}\right) \mathrm{Cl}\right]$, para $\delta-286$ no complexo. De acordo com estes resultados, a hapticidade vai de $\eta^{5}$ no composto de partida para uma média entre $\eta^{1}$ e $\eta^{3}$ no referido derivado. Tamanha mudança ainda não foi suficiente para ser observada no espectro de $\mathrm{RMN}$ de ${ }^{13} \mathrm{C}$ que novamente não revelou diferença entre carbonos ciclopentadienílicos.

Devido à sua instabilidade frente a reações de redistribuição ${ }^{20}$, compostos heterolépticos de $\mathrm{Sn}(\mathrm{II})$ contendo apenas um anel Cp são bastante raros. O primeiro exemplar a ser isolado e caracterizado por difração de raios-X, $\left[\mathrm{Sn}\left(\eta^{5}-\mathrm{C}_{5} \mathrm{H}_{5}\right) \mathrm{N}=\mathrm{C}\left(\mathrm{NMe}_{2}\right)_{2}\right]$, foi obtido reagindo-se $\left[\mathrm{Sn}\left(\eta^{5}-\mathrm{C}_{5} \mathrm{H}_{5}\right)_{2}\right]$ com $\mathrm{LiN}=\mathrm{C}\left(\mathrm{NMe}_{2}\right)_{2}$ (ii). Nesta reação ocorreu a eliminação de $\operatorname{Li}\left(\eta^{5}-\mathrm{C}_{5} \mathrm{H}_{5}\right)$.

Quando $\left[\mathrm{Sn}\left(\eta^{5}-\mathrm{C}_{5} \mathrm{H}_{5}\right)_{2}\right]$ é tratado com $\mathrm{LiBu}^{\mathrm{n}}$ em thf numa temperatura de $-78^{\circ} \mathrm{C}$ obtém-se a espécie, $\left[\mathrm{Sn}\left(\eta^{5}-\mathrm{C}_{5} \mathrm{H}_{4} \mathrm{Li}\right)_{2}\right]$. Ao reagi-lo com $\mathrm{P}\left(\mathrm{NPr}_{2}{ }_{2}\right)_{2} \mathrm{Cl}$ ocorre a produção do estanoceno $\left[\mathrm{Sn}\left(\eta^{5}-\mathrm{C}_{5} \mathrm{H}_{4} \mathrm{P}\left(\mathrm{NPr}_{2}^{\mathrm{i}}\right)_{2}\right\}_{2}\right]$ (Figura 5).

Este derivado apresenta um interessante potencial de atuar como ligante bi- ou tetradentado na preparação de complexos com metais de transição, uma vez que existem átomos de fósforo ou nitrogênio com pares de elétrons disponíveis podendo atuar como centros doadores da molécula.

De maneira semelhante ao $\left[\mathrm{Sn}\left(\eta^{5}-\mathrm{C}_{5} \mathrm{H}_{5}\right)_{2}\right]$, seu parente metilado $\left[\mathrm{Sn}\left(\eta^{5}-\mathrm{Cp}\right)_{2}\right] \quad\left\{\mathrm{Cp}^{*}=\mathrm{C}_{5} \mathrm{Me}_{5}^{-}\right\}$também reage com $\mathrm{SnCl}_{2}, \mathrm{HBF}_{4}$ e $\mathrm{HOSO}_{2} \mathrm{CF}_{3} /$ piridine ou bipiridine produzindo espécies com apenas um anel $\mathrm{Cp}$ tais como $\left[\mathrm{Sn}\left(\eta^{5}-\mathrm{Cp}^{*}\right) \mathrm{Cl}\right]$, $\left[\operatorname{Sn}\left(\eta^{5}-\mathrm{Cp}^{*}\right)\left(\mathrm{BF}_{4}\right)\right],\left[\operatorname{Sn}\left(\eta^{5}-\mathrm{Cp}^{*}\right)(\mathrm{py})\left(\mathrm{OSO}_{2} \mathrm{CF}_{3}\right)\right]$ e $\left[\operatorname{Sn}\left(\eta^{5}-\right.\right.$ $\mathrm{Cp} *)$ (bipy) $\left.\left(\mathrm{OSO}_{2} \mathrm{CF}_{3}\right)\right]$, (Esquema 2$)$.

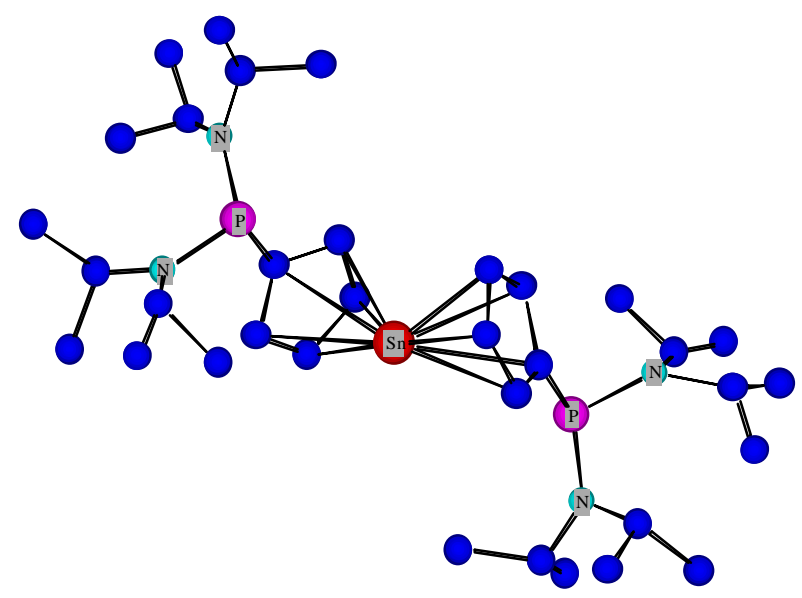

Figura 5. Estrutura molecular de $\left[S n\left\{\eta^{5}-C_{5} H_{4} P\left[N\left(\operatorname{Pr}^{i}\right)_{2}\right]\right\}_{2}\right]$.

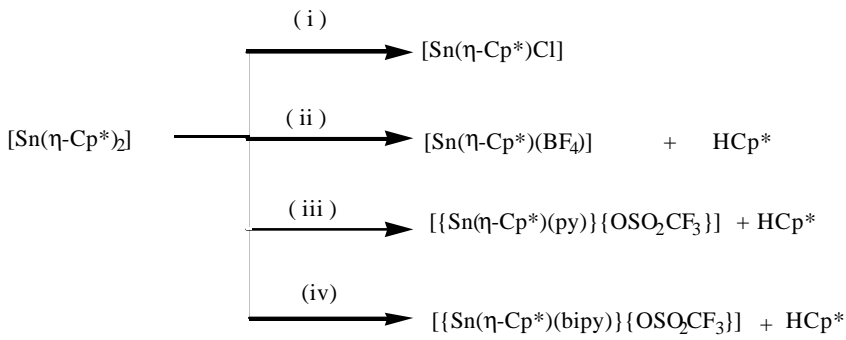

Condições

(i) $\mathrm{SnCl}_{2}{ }^{22}$; (ii) $\mathrm{HBF}_{4} / \mathrm{Et}_{2} \mathrm{O}^{23}$;

(iii) $\mathrm{HOSO}_{2} \mathrm{CF}_{3} / \mathrm{CH}_{2} \mathrm{Cl}_{2} / \mathrm{py}^{24}$;

(iv) $\mathrm{HOSO}_{2} \mathrm{CF}_{3} / \mathrm{CH}_{2} \mathrm{Cl}_{2} / \mathrm{bipy}^{24}$

Esquema 2. Reações do composto [ $\left.\operatorname{Sn}\left(\boldsymbol{\eta}^{5}-C p^{*}\right) 2\right]$.

De modo análogo $\left[\mathrm{Sn}\left(\eta^{5}-\mathrm{Cp}^{\mathrm{s}}\right)_{2}\right]$ reage com $\mathrm{SnCl}_{2}{ }^{22}, \mathrm{HBF}_{4}$ gerando compostos estanosos monociclopentadienílicos, (Esquema 3).

Ao se adicionar $\mathrm{HOSO}_{2} \mathrm{CF}_{3}$ à uma solução de $\left[\operatorname{Sn}\left(\eta^{5}-\mathrm{Cp}^{\mathrm{s}}\right)_{2}\right]$ em tolueno, ocorre a eliminação de HCps como esperado. Entretanto na ausência de bases coordenantes como piridina ou bipiridina o composto estanoso obtido existe no estado sólido como um par iônico cujos íons constituintes são $\operatorname{Sn}\left(\eta^{5}-\mathrm{Cp}^{\mathrm{s}}\right)^{+}$e $\left\{\mathrm{Sn}\left(\eta^{5}-\mathrm{Cp}^{\mathrm{s}}\right)\left(\mathrm{OSO}_{2} \mathrm{CF}_{3}\right)_{2}\right\}^{-}$, e não $\mathrm{Sn}\left(\eta^{5}-\mathrm{Cp}^{\mathrm{s}}\right)^{+}$e ${ }^{-} \mathrm{OSO}_{2} \mathrm{CF}_{3}$ como esperado. A presença de dois centros metálicos de $\mathrm{Sn}(\mathrm{II})$ foi revelada por difração de raios-X e confirmada por resso- 


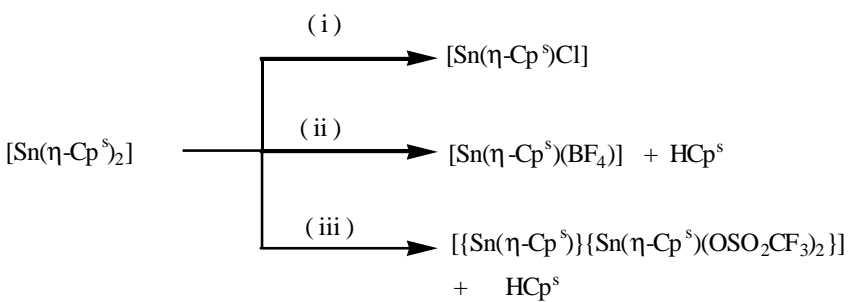

Condições:

(i) $\mathrm{SnCl}_{2}{ }^{21}$ (ii) $\mathrm{HBF}_{4} / \mathrm{Et}_{2} \mathrm{O}^{25}$;

(iii) $\mathrm{HOSO}_{2} \mathrm{CF}_{3}$ /tolueno 25

Esquema 3. Reações do composto [Sn( $\left.\left(\eta^{5}-\mathrm{Cp}^{s}\right)_{2}\right]$.

nância magnética nuclear de ${ }^{119} \mathrm{Sn}$ no estado sólido bem como por espectroscopia Mössbauer de ${ }^{119} \mathrm{Sn}$.

A reação subseqüente do derivado $\mathrm{Sn}\left(\eta^{5}-\mathrm{Cp}^{\mathrm{S}}\right) \mathrm{Cl}$ (i) com $\mathrm{SnCl}_{2}$ em thf levou à formação de uma espécie poliiônica de formulação $\left[\mathrm{Sn}_{9}\left(\eta^{5}-\mathrm{Cp}^{\mathrm{S}}\right)_{6} \mathrm{Cl}_{12}\right]$, (Figura 6), a qual é formada pela agregação de seis cátions $\operatorname{Sn}\left(\eta^{5}-\mathrm{Cp}^{\mathrm{S}}\right)^{+}$com dois tipos de ânions, um de formulação $\mathrm{SnCl}_{6}{ }^{4-}$ e dois outros de formulação $\mathrm{SnCl}_{3}{ }^{-27}$.
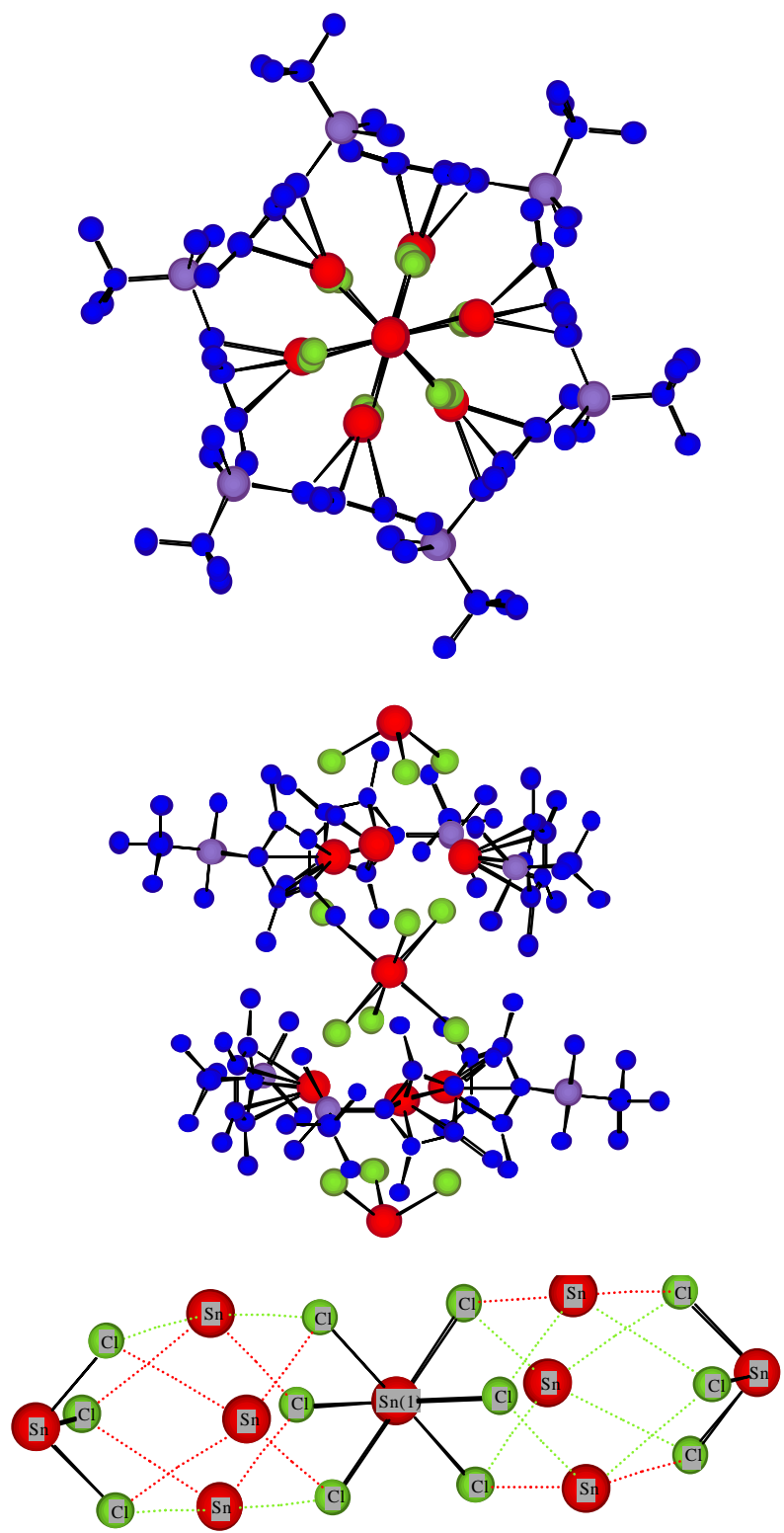

Figura 6. Estrutura molecular do composto $\left[\mathrm{Sn}_{9}\left(\eta^{5}-\mathrm{CpS}\right)_{6} \mathrm{Cl}_{12}\right]$.
As propriedades gerais dos estanocenos podem ser enumeradas de forma resumida da seguinte forma (i) estabilidade e propriedades estruturais estão diretamente relacionadas com a natureza do grupo ciclopentadienílico. Grupos volumosos geram compostos estáveis e com anéis paralelos e grupos menos volumosos formam derivados sensíveis ao ar e umidade e com anéis não paralelos. (ii) De acordo com estudos por difração de raios-X o $\mathrm{Sn}$ (II) se liga de forma assimétrica ao anel $\mathrm{Cp}$. Experimentos de $\mathrm{RMN}$ de ${ }^{13} \mathrm{C}$ e ${ }^{119} \mathrm{Sn}$ não revelam diferença química nessas ligações somente quando um dos aneis Cp é substituído por outros grupos, ver Esquemas 2 e 3, é que se observa uma drástica mudança no deslocamento químico do ${ }^{119} \mathrm{Sn}$ sem reflexo, no entanto, no de ${ }^{13} \mathrm{C}$. Finalmente (iii) estanocenos podem ser utilizados com sucesso como material de partida na preparação de outros compostos semelhantes.

\section{LISTA DE ABREVIAÇÕES}

bipy
$\mathrm{Bu}^{\mathrm{t}}$
$\mathrm{Bz}$
$\mathrm{Cp}$

denota o deslocamento químico no espectro de RMN no estado sólido 


\section{REFERÊNCIAS}

1. (a) Fischer, E. O.; Grubert, H. Z.; Naturforsch 1956, B11, 423; (b) Atwood, J. L.; Hunter, W. E.; Cowley, A. H.; Jones, R. A.; Stewart, C. A.; J. Chem. Soc., Chem. Commun. 1981, 925; (c) Panattoni, C.; Bombieri, G.; Croatto, U.; Acta Cryst., Section C. 1966, 21, 823; (d) Almenningen, A.; Haaland, A.; Motzfeldt, T.; J. Organomet. Chem. 1967, 97, 7; (e) Wrackmeyer, B.; Kupce, E.; Kehr, G.; Sebald, A.; Magn. Reson. Chem. 1992, 30, 964.

2. Neumann, P.; Chem. Rev 1991, 91, 311.

3. Wilkinson, G.; Stone, F. G. A.; Abel, E. W.; eds, Comprehensive Organometallic Chemistry, $1^{\mathrm{a}}$ edição, Pergamon Press, Oxford, 1982, 3, 1043.

4. Janiak, C.; Schumann, H.; Hahn, E.; Heeg, M.; Kolax, J. C.; Loebel, J.; Rausch, M. D.; Zuckerman, J. J.; Chem. Ber. 1986, 119, 2656.

5. Burkey, D. J.; Hanusa, T. P.; Organometallics 1995, 14, 11.

6. (a) Jutzi, P.; Kohl, F.; Hofmann, P.; Krüger, C.; Tsay, Y. -H.; Chem. Ber. 1980, 113, 757; (b) Wrackmeyer, B.; Merwin, L. H.; Sebald, A.; Magn. Reson. Chem. 1991, 29, 260.

7. (a) Huheey, J. E.; Keiter, E. A.; Keiter, R. L.; Inorganic Chemistry: Principles of Structure and Reactivit, $4^{\mathrm{a}}$ edição, Harper Collins College Publishers, 1993, 213; (b) Lawton, S. L.; Jacobson, R. A.; Inorg. Chem. 1966, 5, 743; Lawton, S. L.; Jacobson, R. A.; Inorg. Chem 1966, 5, 743; (c) Mahjoub, A. R.; Hoser, A.; Fuchs, J.; Seppelt, K.; Angew. Chem., Int. Ed. Engl. 1989, 28, 1526

8. Williams, R. A.; Hanusa, T. P.; Huffman, J. C.; Organometallics 1990, 9, 1128; Williams, R. A.; Hanusa, T. P.; Tesh, K. F.; J. Am. Chem. Soc. 1991, 113, 4843,.

9. (a) Heeg, M. J.; Janiak, C.; Zuckerman, J. J.; J. Am. Chem. Soc. 1984, 106, 4259. (b) Heeg, M. J.; Herber, R. H.; Janiak, C.; Zuckerman, J. J.; Schumann, H.; Manders, W. F.; J. Organomet. Chem. 1988, 346, 321. (c) Janiak, C.; Schumann, H.; Stader, C.; Wrackmeyer, B.; Zuckerman, J. J.; Chem. Ber. 1988, 121, 1745.

10. Constantine, S. P.; Hitchcock, P. B.; Lawless, G. A.; de Lima, G. M.; J. Chem. Soc., Chem. Commun. 1996, 1101.

11. Wadepohl, H.; Pritzkow, H.; Siebert, W.; Organometallics 1983, 2, 1899.
12. Schmid, G.; Zaika, D.; Boese, R.; Angew. Chem., Int. Ed. Engl. 1985, 24, 602.

13. Kuhn, N.; Henkel, G.; Stubenrauch, S.; J. Chem. Soc., Chem. Commun. 1992, 760.

14. (a) Bos, K. D.; Bulten, E. J.; Noltes, J. G.; J. Organomet. Chem. 1972, 39, C52; (b) Bos, K. D.; Bulten, E. J.; Noltes, J. G.; Spek, A. L.; J. Organomet. Chem. 1975, 99, 71.

15. Stalke, D.; Paver, M. A.; Wright, D. S.; Angew. Chem., Int. Ed. Engl. 1993, 12, 1.

16. Davidson, M. G.; Stalke, D.; Wright, D. S.; Angew. Chem., Int. Ed. Engl. 1992, 31, 1226.

17. Paver, M. A.; Russel, C. A.; Stalke, D.; Wright, D. S.; Inorg. Chem. 1994, 33, 2370.

18. Dory, T. S.; Zuckerman, J. J.; J. Organomet. Chem. 1985, $281, \mathrm{C} 1$.

19. Veith, M.; Mathur, C.; Huch, V.; Organometallics 1996, $15,2858$.

20. Compostos heterolépticos de $\operatorname{Sn}(\mathrm{II}), \operatorname{Sn}\left(\eta^{5}-\mathrm{Cp}\right) \mathrm{R}$ tendem se redistribuir gerando $\mathrm{SnCp}_{2}$ e $\mathrm{SnR}_{2}$, desta forma se tornam de difícil preparação.

21. Cowley, A. H.; Lasch, J. G.; Norman, N. C.; Stewart, C. A.; Wright, T. C.; Organometallics 1983, 2, 1691.

22. Constantine, S. P.; de Lima, G. M.; Hitchcock, P. B.; Keates, J. M.; Lawless, G. A.; Marziano, I.; Organometallics 1997, 16, 793

23. (a) Jutzi, P.; Kohl, F.; Hofmann, P.; Krüger, C.; Tsay, Y. -H.; Chem. Ber. 1980, 113, 757; (b) Jutzi, P.; Kohl, F.; Krüger, C.; Angew. Chem., Int. Ed. Engl. 1979, 18, 59.

24. (a) Jutzi, P.; Kohl, F. X.; Krüger, C.; Wolmershauser, G.; Hofmann, P.;Stauffert, P.; Angew. Chem., Int. Ed. Engl. 1982, 21, 70; (b) Kohl, F. X.; Schluter, E.; Jutzi, P.; Krüger, C.; Wolmershauser, G.; Hofmann, P.; Stauffert, P.; Chem. Ber. 1984, 117, 1178.

25. de Lima, G. M.; D. Phil Thesis, University of Sussex 1997.

26. de Lima, G. M., Pierssens, L. J. -M.; Mahieu, B.; Hyperfine Interactions 1999, 122, 327.

27. Constantine, S. P.; de Lima, G. M.; Hitchcock, P. B.; Keates, J. M.; Lawless, G. A.; J. Chem. Soc., Chem. Commun. 1996, 2337.

28. Cowley, A. H.; Lasch, J. G.; Norman, N. C.; Schluter, E.; Angew. Chem., Int. Ed. Engl. 1984, 23, 616. 